\title{
An Extension of Modular Sequence Spaces
}

\author{
Hemen Dutta ${ }^{1}$ and Iqbal H. Jebril ${ }^{2}$ \\ ${ }^{1}$ Department of Mathematics, Gauhati University, Guwahati, Assam 781014, India \\ ${ }^{2}$ Department of Mathematics, Faculty of Science, Taibah University, P.O. Box 30097, Almadinah Almunawwarah 41477, Saudi Arabia
}

Correspondence should be addressed to Iqbal H. Jebril; iqbal501@hotmail.com

Received 1 April 2013; Accepted 2 June 2013

Academic Editor: Jinde Cao

Copyright (C) 2013 H. Dutta and I. H. Jebril. This is an open access article distributed under the Creative Commons Attribution License, which permits unrestricted use, distribution, and reproduction in any medium, provided the original work is properly cited.

The main aim of this paper is to present an extension of the modular sequence spaces by means of Cesàro mean of order one, to investigate several relevant algebraic and topological properties, and derive some other spaces in the sequel.

\section{Introduction}

Throughout the paper $w(X)$ will represent the spaces of all $X$ valued sequences spaces, where $(X, q)$ is a seminormed space, seminormed by $q$. For $X=C$, the space of complex numbers, these represent the corresponding scalar valued sequence spaces. The zero sequence is denoted by $\bar{\theta}=(\theta, \theta, \theta, \ldots)$, where $\theta$ is the zero element of $X$.

An Orlicz function is a function $M:[0, \infty) \rightarrow[0, \infty)$, which is continuous, nondecreasing, and convex with $M(0)=$ $0, M(x)>0$, for $x>0$ and $M(x) \rightarrow \infty$, as $x \rightarrow \infty$.

If convexity of Orlicz function $M$ is replaced by $M(x+$ $y) \leq M(x)+M(y)$, then this function is called a modulus function introduced by Nakano [1].

Lindenstrauss and Tzafriri [2] used the idea of Orlicz function to construct sequence space

$$
\ell_{M}=\left\{x \in w: \sum_{k=1}^{\infty} M\left(\frac{\left|x_{k}\right|}{\rho}\right)<\infty \text {, for some } \rho>0\right\} .
$$

The space $\ell_{M}$ becomes a Banach space, with the norm

$$
\|x\|=\inf \left\{\rho>0: \sum_{k=1}^{\infty} M\left(\frac{\left|x_{k}\right|}{\rho}\right) \leq 1\right\}
$$

which is called an Orlicz space. The space $\ell_{M}$ is closely related to the space $\ell_{p}$ which is an Orlicz sequence space with $M(x)=$ $x^{p}$ for $1 \leq p<\infty$.
Another generalization of Orlicz sequence spaces is due to Woo [3]. Let $\left\{M_{k}\right\}$ be a sequence of Orlicz functions. Define the vector space $\ell\left\{M_{k}\right\}$ by

$$
\ell\left\{M_{k}\right\}=\left\{x \in w: \sum_{k=1}^{\infty} M_{k}\left(\frac{\left|x_{k}\right|}{\rho}\right)<\infty \text {, for some } \rho>0\right\}
$$

and equip this space with the norm

$$
\|x\|=\inf \left\{\rho>0: \sum_{k=1}^{\infty} M_{k}\left(\frac{\left|x_{k}\right|}{\rho}\right) \leq 1\right\} .
$$

Then $\ell\left\{M_{k}\right\}$ becomes a Banach space and is called a modular sequence space. The space $\ell\left\{M_{k}\right\}$ also generalizes the concept of modular sequence space introduced earlier by Nakano [4], who considered the space $\ell\left\{M_{k}\right\}$ when $M_{k}(x)=$ $x^{\alpha_{k}}$, where $1 \leq \alpha_{k}<\infty$ for $k \geq 1$.

An Orlicz function $M$ is said to satisfy the $\Delta_{2}$-condition for all values of $u$, if there exists a constant $K>0$, such that $M(2 u) \leq k M(u)(u \geq 0)$. The $\Delta_{2}$-condition is equivalent to the satisfaction of inequality $M(l u) \leq k l u M(u)$ for all values of $u$ and for $l>1$ (see [5]).

The previous $\Delta_{2}$-condition implies $M(l u) \leq$ $K l^{\log _{2} K} M(u)$, for all $u>0, l>1$.

Bektaş and Altin [6], Parasar and Choudhary [7], Mursaleen et al. [8], Dutta and Başar [9], Dutta and Bilgin [10], Karakaya and Dutta [11], Tripathy and Dutta [12], Jebril [13], and many others have studied different summable spaces and other sequence spaces using Orlicz functions. 
A $B K$-space (introduced by Zeller [14]) $(X,\|\cdot\|)$ is a Banach space of complex sequences $x=\left(x_{k}\right)$ in which the coordinate maps are continuous; that is, $\left|x_{k}^{n}-x_{k}\right| \rightarrow 0$, whenever $\left\|x^{n}-x\right\| \rightarrow 0$ as $n \rightarrow \infty$, where $x^{n}=\left(x_{k}^{n}\right)$, for all $n \in N$ and $x=\left(x_{k}\right)$.

Let $A$ denote the set of all complex sequences which have only a finite number of nonzero coordinates, and let $\lambda$ denote a $B K$-space of sequences $x=\left(x_{k}\right)$ which contains $A$. An element $x=\left(x_{k}\right)$ of $\lambda$ will be called sectionally convergent if

$$
x^{(n)}=\sum_{k=1}^{n} x_{k} e_{k} \longrightarrow x, \quad \text { as } n \longrightarrow \infty,
$$

where $e_{k}=\left(\delta_{k i}\right)$, where $\delta_{k k}=1, \delta_{k i}=0$ for $k \neq i$.

$\lambda$ will be called $A K$-space if and only if each of its elements is sectionally convergent.

Let $\mathbf{M}=\left(M_{k}\right)$ be a sequence of Orlicz functions, let $X$ be a seminormed space with seminorm $q$, let $p=\left(p_{k}\right)$ be sequence of positive real numbers, and let $C=\left(c_{n k}\right)_{n, k=0}^{\infty}$ be the Cesàro matrix of order one with $c_{n k}=1 /(n+1)$ if $0 \leq k \leq n$ and $c_{n k}=0$, otherwise. Then for nonnegative real numbers $s$ we define

$$
\begin{aligned}
& \ell\left\{M_{k}, p, q, s, C\right\} \\
& =\left\{x \in w(X): \sum_{k=0}^{\infty} k^{-s}\left[M_{k}\left(q\left(\frac{\sum_{j=0}^{k} x_{j}}{\rho(k+1)}\right)\right)\right]^{p_{k}}\right. \\
& <\infty, \text { for some } \rho>0\} .
\end{aligned}
$$

The following inequality will be used throughout the paper. Let $p=\left(p_{k}\right)$ be a positive sequence of real numbers with $0<p_{k} \leq \sup p_{k}=G, D=\max \left\{1,2^{G-1}\right\}$. Then for all $a_{k}, b_{k} \in C$ for all $k \in N$, we have

$$
\left|a_{k}+b_{k}\right|^{p_{k}} \leq D\left\{\left|a_{k}\right|^{p_{k}}+\left|b_{k}\right|^{p_{k}}\right\}
$$

\section{Main Results}

In this section we give the theorems that characterize the structure of the class of sequences $\ell\left\{M_{k}, p, q, s, C\right\}$ and some other spaces which can be derived from this space.

Theorem 1. Let $p=\left(p_{k}\right)$ be bounded sequence of positive reals; then $\ell\left\{M_{k}, p, q, s, C\right\}$ is a linear space over the field of complex numbers.

Proof. Let $x, y \in \ell\left\{M_{k}, p, q, s, C\right\}$ and $\alpha, \beta \in C$. Then there exist some $\rho_{1}>0$ and $\rho_{2}>0$ such that

$$
\begin{aligned}
& \sum_{k=0}^{\infty} k^{-s}\left[M_{k}\left(q\left(\frac{\sum_{j=0}^{k} x_{j}}{\rho_{1}(k+1)}\right)\right)\right]^{p_{k}}<\infty, \\
& \sum_{k=0}^{\infty} k^{-s}\left[M_{k}\left(q\left(\frac{\sum_{j=0}^{k} y_{j}}{\rho_{2}(k+1)}\right)\right)\right]^{p_{k}}<\infty .
\end{aligned}
$$

We consider $\rho_{3}=\max \left(2|\alpha| \rho_{1}, 2|\beta| \rho_{2}\right)$. Since each $M_{k}$ is nondecreasing and convex, and since $q$ is a seminorm,

$$
\begin{aligned}
& \sum_{k=0}^{\infty} k^{-s}\left[M_{k}\left(q\left(\frac{\sum_{j=0}^{k}\left(\alpha x_{k}+\beta y_{k}\right)}{\rho_{3}(k+1)}\right)\right)\right]^{p_{k}} \\
& \leq \sum_{k=0}^{\infty} k^{-s}\left[M _ { k } \left(q\left(\frac{\sum_{j=0}^{k}\left(\alpha x_{j}\right)}{\rho_{3}(k+1)}\right)\right.\right. \\
& \left.\left.+q\left(\frac{\sum_{j=0}^{k}\left(\beta y_{j}\right)}{\rho_{3}(k+1)}\right)\right)\right]^{p_{k}} \\
& \leq \sum_{k=0}^{\infty} k^{-s}\left[M _ { k } \left(q\left(\frac{\sum_{j=0}^{k} x_{j}}{2 \rho_{1}(k+1)}\right)\right.\right. \\
& \left.\left.+q\left(\frac{\sum_{j=0}^{k} y_{j}}{2 \rho_{2}(k+1)}\right)\right)\right]^{p_{k}} \\
& \leq D\left\{\sum_{k=0}^{\infty} k^{-s}\left[M_{k}\left(q\left(\frac{\sum_{j=0}^{k} x_{j}}{\rho_{1}(k+1)}\right)\right)\right]^{p_{k}}\right. \\
& \left.+\sum_{k=0}^{\infty} k^{-s}\left[M_{k}\left(q\left(\frac{\sum_{j=0}^{k} y_{j}}{\rho_{2}(k+1)}\right)\right)\right]^{p_{k}}\right\}<\infty .
\end{aligned}
$$

This completes the proof.

Theorem 2. $\ell\left\{M_{k}, p, q, s, C\right\}$ is a paranormed space (need not total paranorm) space with paranorm $g$, defined as follows

$$
\begin{gathered}
g(x)=\inf \left\{\rho^{p_{n} / H}: \sum_{k=0}^{\infty} k^{-s}\left[M_{k}\left(q\left(\frac{\sum_{j=0}^{k} x_{j}}{\rho(k+1)}\right)\right)\right]\right. \\
\leq 1, n=1,2, \ldots\},
\end{gathered}
$$

where $H=\max \left\{1, \sup _{k} p_{k}\right\}$.

Proof. Clearly $g(x)=g(-x)$. Since $M_{k}(0)=0$, for all $k \in N$ we get $\inf \left\{\rho^{p_{n} / H}\right\}=0$ for $x=\theta$.

Now let $x, y \in \ell\left\{M_{k}, p, q, s, C\right\}$, and let us choose $\rho_{1}>0$ and $\rho_{2}>0$ such that

$$
\begin{gathered}
\sum_{k=0}^{\infty} k^{-s}\left[M_{k}\left(q\left(\frac{\sum_{j=0}^{k} x_{j}}{\rho_{1}(k+1)}\right)\right)\right] \leq 1, \\
\sum_{k=0}^{\infty} k^{-s}\left[M_{k}\left(q\left(\frac{\sum_{j=0}^{k} y_{j}}{\rho_{2}(k+1)}\right)\right)\right] \leq 1 .
\end{gathered}
$$


Let $\rho=\rho_{1}+\rho_{2}$. Then we have

$$
\begin{aligned}
\sum_{k=0}^{\infty} k^{-s} & {\left[M_{k}\left(q\left(\frac{\sum_{j=0}^{k}\left(x_{j}+y_{j}\right)}{\rho(k+1)}\right)\right)\right] } \\
\leq & \left(\frac{\rho_{1}}{\rho_{1}+\rho_{2}}\right) \sum_{k=0}^{\infty} k^{-s}\left[M_{k}\left(q\left(\frac{\sum_{j=0}^{k} x_{j}}{\rho_{1}(k+1)}\right)\right)\right] \\
& +\left(\frac{\rho_{2}}{\rho_{1}+\rho_{2}}\right) \sum_{k=0}^{\infty} k^{-s}\left[M_{k}\left(q\left(\frac{\sum_{j=0}^{k} y_{j}}{\rho_{2}(k+1)}\right)\right)\right] \leq 1 .
\end{aligned}
$$

Hence $g(x+y) \leq g(x)+g(y)$.

Finally let $\lambda$ be a given non-zero scalar; then the continuity of the scalar multiplication follows from the following equality

$g(\lambda x)$

$$
\begin{gathered}
=\inf \left\{\rho^{p_{n} / H}: \sum_{k=0}^{\infty} k^{-s}\left[M_{k}\left(q\left(\frac{\sum_{j=0}^{k}\left(\lambda x_{j}\right)}{\rho(k+1)}\right)\right)\right]\right. \\
\leq 1, n=1,2, \ldots\} \\
=\inf \left\{(|\lambda| s)^{p_{n} / H}: \sum_{k=0}^{\infty} k^{-s}\left[M_{k}\left(q\left(\frac{\sum_{j=0}^{k} x_{j}}{s(k+1)}\right)\right)\right]\right. \\
\leq 1, n=1,2, \ldots\}, \quad \text { where } s=\frac{\rho}{|s|} .
\end{gathered}
$$

This completes the proof.

The proof of the following theorem is easy, so omitted.

Theorem 3. Let $\mathbf{M}=\left(M_{k}\right)$ and $\mathbf{T}=\left(T_{k}\right)$ be sequences of Orlicz functions. For any two sequences $p=\left(p_{k}\right)$ and $t=\left(t_{k}\right)$ of bounded positive real numbers and for any two seminorms $q_{1}$ and $q_{2}$ one has

(i) if $q_{1}$ is stronger than $q_{2}$, then $\ell\left\{M_{k}, p, q_{1}, s, C\right\} \subset$ $\ell\left\{M_{k}, p, q_{2}, s, C\right\}$,

(ii) $\ell\left\{M_{k}, p, q_{1}, s, C\right\} \cap \ell\left\{M_{k}, p, q_{2}, s, C\right\} \subset \ell\left\{M_{k}, p, q_{1}+\right.$ $\left.q_{2}, s, C\right\}$,

(iii) $\ell\left\{M_{k}, p, q, s, C\right\} \cap \ell\left\{T_{k}, p, q, s, C\right\} \quad \subset \quad \ell\left\{M_{k}+\right.$ $\left.T_{k}, p, q, s, C\right\}$,

(iv) $\ell\left\{M_{k}, p, q_{1}, s, C\right\} \cap \ell\left\{M_{k}, t, q_{2}, s, C\right\} \neq \varphi$,

(v) If $s_{1} \leq s_{2}$, then $\ell\left\{M_{k}, p, q, s_{1}, C\right\} \subset \ell\left\{M_{k}, p, q, s_{2}, C\right\}$.

Theorem 4. Let $\mathbf{M}=\left(M_{k}\right)$ and $\mathbf{T}=\left(T_{k}\right)$ be sequences of Orlicz functions which satisfy $\Delta_{2}$-condition and $s>1$, then

$$
\ell\left\{M_{k}, p, q, s, C\right\} \subseteq \ell\left\{T_{k} \circ M_{k}, p, q, s, C\right\} .
$$

Proof. Let $x \in \ell\left\{M_{k}, p, q, s, C\right\}$ and $\varepsilon>0$. We choose $0<$ $\delta<1$ such that each $M_{k}(u)<\varepsilon$ for $0 \leq u \leq \delta$. We write $y_{k}=M_{k}\left(q\left(\left(\sum_{j=0}^{k} x_{j}\right) / \rho(k+1)\right)\right)$ and consider

$$
\sum_{k=0}^{\infty} k^{-s}\left[T_{k}\left(y_{k}\right)\right]^{p_{k}}=\sum_{1} k^{-s}\left[T_{k}\left(y_{k}\right)\right]^{p_{k}}+\sum_{2} k^{-s}\left[T_{k}\left(y_{k}\right)\right]^{p_{k}}
$$

where the first summation is over $y_{k} \leq \delta$ and the second is over $y_{k}>\delta$. Now we have

$$
\sum_{1} k^{-s}\left[T_{k}\left(y_{k}\right)\right]^{p_{k}}<\max \left(1, \varepsilon^{H}\right) \sum_{k=1}^{\infty} k^{-s}<\infty, \quad \text { since } s>1 \text {. }
$$

For $y_{k}>\delta$, we use the fact that

$$
y_{k}<\frac{y_{k}}{\delta} \leq 1+\left(\frac{y_{k}}{\delta}\right)
$$

Since each $T_{k}$ is non-decreasing and convex, it follows that

$$
\begin{array}{r}
T_{k}\left(y_{k}\right)<T_{k}\left(1+\frac{y_{k}}{\delta}\right)<\frac{1}{2} T_{k}(2)+\frac{1}{2} T_{k}\left(2 \frac{y_{k}}{\delta}\right), \\
\text { for each } k \text { in } N .
\end{array}
$$

Since each $T_{k}$ satisfies $\Delta_{2}$-condition, we have

$$
T_{k}\left(y_{k}\right)<\frac{1}{2} K \frac{y_{k}}{\delta} T_{k}(2)+\frac{1}{2} K \frac{y_{k}}{\delta} T_{k}(2)=K y_{k} \delta^{-1} T_{k}(2) .
$$

Hence

$$
\begin{aligned}
\sum_{2} k^{-s}\left[T_{k}\left(y_{k}\right)\right]^{p_{k}} \leq & \max \left(1,\left(K \delta^{-1} M(2)\right)^{H}\right) \\
& \times \sum_{k=1}^{\infty} k^{-s}\left(y_{k}\right)^{p_{k}}<\infty .
\end{aligned}
$$

Thus

$$
\begin{aligned}
\sum_{k=0}^{\infty} k^{-s}\left[T_{k}\left(y_{k}\right)\right]^{p_{k}}= & \sum_{1} k^{-s}\left[T_{k}\left(y_{k}\right)\right]^{p_{k}}+\sum_{2} k^{-s}\left[T_{k}\left(y_{k}\right)\right]^{p_{k}} \\
\leq & \max \left(1, \varepsilon^{H}\right) \\
& \times \sum_{k=1}^{\infty} k^{-s}+\max \left(1,\left(K \delta^{-1} M(2)\right)^{H}\right) \\
& \times \sum_{k=1}^{\infty} k^{-s}\left(y_{k}\right)^{p_{k}}<\infty .
\end{aligned}
$$

Hence $x \in \ell\left\{T_{k} \circ M_{k}, p, q, s, C\right\}$.

This completes the proof. 
Taking $M_{k}(x)=x$, for all $x \in[0, \infty)$ and $k$ in $N$, in Theorem 4 , we get the next corollary.

Corollary 5. Let $\mathbf{M}=\left(M_{k}\right)$ be any sequence of Orlicz functions which satisfy $\Delta_{2}$-condition and $s>1$, then

$$
\ell\{p, q, s, C\} \subseteq \ell\left\{M_{k}, p, q, s, C\right\} .
$$

We will write $f \approx g$ for non-negative functions $f$ and $g$ whenever $C_{1} f \leq g \leq C_{2} f$ for some $C_{j}>0, j=1,2$.

Theorem 6. Let $\mathbf{M}=\left(M_{k}\right)$ and $\mathbf{T}=\left(T_{k}\right)$ be sequences of Orlicz functions. If $M_{k} \approx T_{k}$ for each $k \in N$, then $\ell\left\{M_{k}, p, q, s, C\right\}=\ell\left\{T_{k}, p, q, s, C\right\}$.

Proof. The proof is obvious.

Theorem 7. Let $\mathbf{M}=\left(M_{k}\right)$ be a sequence of Orlicz functions. If $\lim _{t \rightarrow 0}\left(M_{k}(t) / t\right)>0$ and $\lim _{t \rightarrow 0}\left(M_{k}(t) / t\right)<\infty$, for each $k \in N$, then $\ell\left\{M_{k}, p, q, s, C\right\}=\ell\{p, q, s, C\}$.

Proof. If the given conditions are satisfied, we have $M_{k}(t) \approx t$ for each $k$, and the proof follows from Theorem 6 .

If we take $s=0$, the sequence space $\ell\left\{M_{k}, p, q, s, C\right\}$ reduces to the following sequence space:

$$
\begin{aligned}
& \ell\left\{M_{k}, p, q, C\right\} \\
& =\left\{x \in w(X): \sum_{k=0}^{\infty}\left[M_{k}\left(q\left(\frac{\sum_{j=0}^{k} x_{j}}{\rho(k+1)}\right)\right)\right]^{p_{k}}\right. \\
& <\infty, \text { for some } \rho>0\} .
\end{aligned}
$$

Theorem 8. Let $p=\left(p_{k}\right)$ be bounded sequence of positive reals, and let $(X, q)$ be a complete seminormed space, then $\ell\left\{M_{k}, p, q, C\right\}$ is a complete paranormed space paranormed by $h$, defined by

$$
\begin{gathered}
h(x)=\inf \left\{\rho^{p_{n} / H}: \sum_{k=0}^{\infty}\left[M_{k}\left(q\left(\frac{\sum_{j=0}^{k} x_{j}}{\rho(k+1)}\right)\right)\right]\right. \\
\leq 1, n=1,2, \ldots\},
\end{gathered}
$$

where $H=\max \left\{1, \sup _{k} p_{k}\right\}$.

Proof. Let $\left(x^{i}\right)$ be a Cauchy sequence in $\ell\left\{M_{k}, p, q, C\right\}$. Let $\delta>$ 0 be fixed, and let $r>0$ be such that for a given $0<\varepsilon<$
$1, \varepsilon / r \delta>0$, and $r \delta \geq 1$. Then there exists a positive integer $n_{0}$ such that

$$
\begin{gathered}
h\left(x^{s}-x^{t}\right)<\frac{\varepsilon}{r \delta}, \quad \forall s, t \geq n_{0} . \\
\Longrightarrow \inf \left\{\rho^{p_{n} / H}: \sum_{k=0}^{\infty}\left[M_{k}\left(q\left(\frac{\sum_{j=0}^{k}\left(x_{j}^{s}-x_{j}^{t}\right)}{\rho(k+1)}\right)\right)\right]\right. \\
\leq 1, n=1,2, \ldots\}<\frac{\varepsilon}{r \delta}, \quad \forall s, t \geq n_{0} .
\end{gathered}
$$

Hence we have

$$
\sum_{k=0}^{\infty}\left[M_{k}\left(q\left(\frac{\sum_{j=0}^{k}\left(x_{j}^{s}-x_{j}^{t}\right)}{h\left(x^{s}-x^{t}\right)(k+1)}\right)\right)\right] \leq 1, \quad \forall s, t \geq n_{0} .
$$

It follows that

$$
\begin{array}{r}
M_{k}\left(q\left(\frac{\sum_{j=0}^{k}\left(x_{j}^{s}-x_{j}^{t}\right)}{h\left(x^{s}-x^{t}\right)(k+1)}\right)\right) \leq 1, \\
\forall s, t \geq n_{0}, \quad k \in N .
\end{array}
$$

For $r>0$ with $M_{k}(r \delta / 2) \geq 1$, we have

$$
\begin{array}{r}
M_{k}\left(q\left(\frac{\sum_{j=0}^{k}\left(x_{j}^{s}-x_{j}^{t}\right)}{h\left(x^{s}-x^{t}\right)(k+1)}\right)\right) \leq M_{k}\left(\frac{r \delta}{2}\right), \\
\forall s, t \geq n_{0}, \quad k \in N .
\end{array}
$$

Since $M_{k}$ is non-decreasing for each $k \in N$, we have

$$
q\left(\frac{\sum_{j=0}^{k}\left(x_{j}^{s}-x_{j}^{t}\right)}{k+1}\right) \leq \frac{r \delta}{2} \cdot \frac{\varepsilon}{r \delta}=\frac{\varepsilon}{2} .
$$

Hence it follows that $\left(x_{k}^{s}\right)$ is a Cauchy sequence in $(X, q)$ for each $k \in N$. But $(X, q)$ is complete, and so $\left(x_{k}^{s}\right)$ is convergent in $(X, q)$ for each $k \in N$.

Let $\lim _{s \rightarrow \infty} x_{k}^{s}=x_{k}$ exists for each $k \in N$.

Now we have for all $s, t \geq n_{0}$,

$$
\begin{gathered}
\inf \left\{\rho^{p_{n} / H}: \sum_{k=0}^{\infty}\left[M_{k}\left(q\left(\frac{\sum_{j=0}^{k}\left(x_{j}^{s}-x_{j}^{t}\right)}{\rho(k+1)}\right)\right)\right]\right. \\
\leq 1, n=1,2, \ldots\}<\varepsilon .
\end{gathered}
$$

Then we have

$$
\begin{array}{r}
\lim _{t \rightarrow \infty}\left\{\operatorname { i n f } \left\{\rho^{p_{n} / H}: \sum_{k=0}^{\infty}\left[M_{k}\left(q\left(\frac{\sum_{j=0}^{k}\left(x_{j}^{s}-x_{j}^{t}\right)}{\rho(k+1)}\right)\right)\right]\right.\right. \\
\leq 1, n=1,2, \ldots\}\}<\varepsilon, \quad \forall s \geq n_{0} .
\end{array}
$$


Using the continuity of Orlicz functions, we have

$$
\begin{gathered}
\inf \left\{\rho^{p_{n} / H}: \sum_{k=0}^{\infty}\left[M_{k}\left(q\left(\frac{\sum_{j=0}^{k}\left(x_{j}^{s}-\lim _{t \rightarrow \infty} x_{j}^{t}\right)}{\rho(k+1)}\right)\right)\right]\right. \\
\leq 1, n=1,2, \ldots\}<\varepsilon, \quad \forall s \geq n_{0} .
\end{gathered}
$$

This implies

$$
\begin{gathered}
\inf \left\{\rho^{p_{n} / H}: \sum_{k=0}^{\infty}\left[M_{k}\left(q\left(\frac{\sum_{j=0}^{k}\left(x_{j}^{s}-x_{j}\right)}{\rho}\right)\right)\right]\right. \\
\leq 1, n=1,2, \ldots\}<\varepsilon, \quad \forall s \geq n_{0} .
\end{gathered}
$$

It follows that $\left(x^{s}-x\right) \in \ell\left\{M_{k}, p, q, C\right\}$.

Since $\left(x^{s}\right) \in \ell\left\{M_{k}, p, q, C\right\}$ and $\ell\left\{M_{k}, p, q, C\right\}$ is a linear space, so we have $x=x^{s}-\left(x^{s}-x\right) \in \ell\left\{M_{k}, p, q, C\right\}$.

This completes the proof.

If we take $s=0$ and $p_{k}=l, a$ constant the sequence space $\ell\left\{M_{k}, p, q, s, C\right\}$ reduces to the following sequence space:

$$
\begin{aligned}
& \ell\left\{M_{k}, q, C\right\} \\
& =\left\{x \in w(X): \sum_{k=0}^{\infty}\left[M_{k}\left(q\left(\frac{\sum_{j=0}^{k} x_{j}}{\rho(k+1)}\right)\right)\right]\right. \\
& <\infty, \text { for some } \rho>0\} .
\end{aligned}
$$

Theorem 9. Let $(X, q)$ be a complete normed space; then $\ell\left\{M_{k}, q, C\right\}$ is a Banach space normed by $\|\cdot\|$, defined by

$$
\|x\|=\inf \left\{\rho: \sum_{k=0}^{\infty}\left[M_{k}\left(q\left(\frac{\sum_{j=0}^{k} x_{j}}{\rho(k+1)}\right)\right)\right] \leq 1\right\} .
$$

Proof. We prove that $\|\cdot\|$ is a norm on $\ell\left\{M_{k}, q, C\right\}$. The completeness part can be proved using similar arguments as applied to prove the previous theorem.

If $x=\theta$, then it is obvious that $\|x\|=0$. Conversely assume $\|x\|=0$. Then using the definition of norm, we have

$$
\inf \left\{\rho: \sum_{k=0}^{\infty}\left[M_{k}\left(q\left(\frac{\sum_{j=0}^{k} x_{j}}{\rho(k+1)}\right)\right)\right] \leq 1\right\}=0 .
$$

This implies that for a given $\varepsilon>0$, there exists some $\rho_{\varepsilon}(0<$ $\left.\rho_{\varepsilon}<\varepsilon\right)$ such that

$$
\sum_{k=0}^{\infty}\left[M_{k}\left(q\left(\frac{\sum_{j=0}^{k} x_{j}}{\rho_{\varepsilon}(k+1)}\right)\right)\right] \leq 1
$$

It follows that

$$
M_{k}\left(q\left(\frac{\sum_{j=0}^{k} x_{j}}{\rho_{\varepsilon}(k+1)}\right)\right) \leq 1, \quad \forall k \in N .
$$

Thus

$$
M_{k}\left(q\left(\frac{\sum_{j=0}^{k} x_{j}}{\varepsilon(k+1)}\right)\right) \leq M_{k}\left(q\left(\frac{\sum_{j=0}^{k} x_{j}}{\rho_{\varepsilon}(k+1)}\right)\right)
$$

$$
\leq 1, \quad \forall k \in N \text {. }
$$

Suppose $\left(\sum_{j=0}^{n_{i}} x_{j}\right) /\left(n_{i}+1\right) \neq 0$, for some $i$. Let $\varepsilon \rightarrow 0$ then $\left(\sum_{j=0}^{n_{i}} x_{j}\right) /\left(\varepsilon\left(n_{i}+1\right)\right) \rightarrow \infty$.

It follows that $M_{k}\left(q\left(\left(\sum_{j=0}^{k} x_{j}\right) /(\varepsilon(k+1))\right)\right) \rightarrow \infty$ as $\varepsilon \rightarrow$ 0 for some $n_{i} \in N$. This is a contradiction.

Therefore $\left(\sum_{j=0}^{k} x_{j}\right) /(k+1)=0$, for all $k \in N$.

It follows that $x_{k}=0$ for all $k \geq 1$. Hence $x=\theta$.

Again proof of the properties $\|x+y\| \leq\|x\|+\|y\|$ and for any scalar $\alpha,\|\alpha x\|=|\alpha|\|x\|$ are similar to that of Theorem 2 .

It is easy to see that $\left\|x^{i}\right\| \rightarrow 0$ implies that $x_{k}^{i} \rightarrow 0$ for each $i \geq 1$. Hence we have the following proposition.

Proposition 10. The space $\ell\left\{M_{k}, q, C\right\}$ is a BK-space.

Now we study the $A K$-characteristic of the space $\ell\left\{M_{k}, q, s, C\right\}$. Before that we give a new definition and prove some results which will be required.

Definition 11. For any sequence of Orlicz functions $\mathbf{M}=$ $\left(M_{k}\right)$, we define

$$
\begin{aligned}
h\left\{M_{k}, q, C\right\}= & \left\{x \in w(X): \sum_{k=0}^{\infty}\left[M_{k}\left(q\left(\frac{\sum_{j=0}^{k} x_{j}}{\rho(k+1)}\right)\right)\right]\right. \\
& <\infty, \text { for every } \rho>0\} .
\end{aligned}
$$

Clearly $h\left\{M_{k}, q, C\right\}$ is a subspace of $\ell\left\{M_{k}, q, C\right\}$. The topology of $h\left\{M_{k}, q, C\right\}$ is the one it inherits from $\|\cdot\|$.

Proposition 12. Let $\mathbf{M}=\left(M_{k}\right)$ be a sequence of Orlicz functions which satisfy $\Delta_{2}$-condition. Then

$$
\ell\left\{M_{k}, q, C\right\}=h\left\{M_{k}, q, C\right\}
$$

Proof. It is enough to prove that $\ell\left\{M_{k}, q, C\right\} \subseteq h\left\{M_{k}, q, C\right\}$.

Let $x \in \ell\left\{M_{k}, q, C\right\}$, then for some $\rho>0$,

$$
\sum_{k=0}^{\infty}\left[M_{k}\left(q\left(\frac{\sum_{j=0}^{k} x_{j}}{\rho(k+1)}\right)\right)\right]<\infty .
$$


Choose an arbitrary $\eta>0$. If $\rho \leq \eta$ then

$$
\begin{gathered}
\sum_{k=0}^{\infty}\left[M_{k}\left(q\left(\frac{\sum_{j=0}^{k} x_{j}}{\eta(k+1)}\right)\right)\right] \\
<\sum_{k=0}^{\infty}\left[M_{k}\left(q\left(\frac{\sum_{j=0}^{k} x_{j}}{\rho(k+1)}\right)\right)\right]<\infty .
\end{gathered}
$$

Let now $\eta<\rho$ and put $l=\rho / \eta>1$.

Since each $M_{k}$ satisfies the $\Delta_{2}$-condition, there exist constants $K_{k}$ such that

$$
\begin{aligned}
\sum_{k=0}^{\infty}\left[M_{k}\left(q\left(\frac{\sum_{j=0}^{k} x_{j}}{\eta(k+1)}\right)\right)\right] \\
\leq \sum_{k=0}^{\infty} K_{k}\left(\frac{\rho}{\eta}\right)^{\log _{2} K_{k}}\left[M_{k}\left(q\left(\frac{\sum_{j=0}^{k} x_{j}}{\rho(k+1)}\right)\right)\right] .
\end{aligned}
$$

Let $S=\sup _{k} K_{k}(\rho / \eta)^{\log _{2} K_{k}}$. Then for every $\eta>0$

$$
\begin{gathered}
\sum_{k=0}^{\infty}\left[M_{k}\left(q\left(\frac{\sum_{j=0}^{k} x_{j}}{\eta(k+1)}\right)\right)\right] \\
\leq S \sum_{k=0}^{\infty}\left[M_{k}\left(q\left(\frac{\sum_{j=0}^{k} x_{j}}{\rho(k+1)}\right)\right)\right]<\infty .
\end{gathered}
$$

This completes the proof.

Proposition 13. Let $(X, q)$ be a complete normed space, then $h\left\{M_{k}, q, C\right\}$ is an AK-space.

Proof. Let $x \in h\left\{M_{k}, q, C\right\}$. Then for each $\varepsilon, 0<\varepsilon<1$, we can find an $s_{0}$ such that

$$
\sum_{k \geq s_{0}}\left[M_{k}\left(q\left(\frac{\sum_{j=0}^{k} x_{j}}{\varepsilon(k+1)}\right)\right)\right] \leq 1
$$

Hence for $s \geq s_{0}$,

$$
\begin{aligned}
\| x & -x^{(s)} \| \\
& =\inf \left\{\rho>0: \sum_{k \geq s+1}\left[M_{k}\left(q\left(\frac{\sum_{j=0}^{k} x_{j}}{\rho(k+1)}\right)\right)\right] \leq 1\right\} \\
& \leq \inf \left\{\rho>0: \sum_{k \geq s}\left[M_{k}\left(q\left(\frac{\sum_{j=0}^{k} x_{j}}{\rho(k+1)}\right)\right)\right] \leq 1\right\}<\varepsilon .
\end{aligned}
$$

Thus we can conclude that $h\left\{M_{k}, q, C\right\}$ is an $A K$ space.

Combining Proposition 10 and Proposition 12, we have the following theorem.
Theorem 14. Let $\mathbf{M}=\left(M_{k}\right)$ be a sequence of Orlicz functions which satisfy $\Delta_{2}$-condition, then $\ell\left\{M_{k}, q, C\right\}$ is an AK-space.

Proposition 15. The space $h\left\{M_{k}, q, C\right\}$ is a closed subspace of $\ell\left\{M_{k}, q, C\right\}$.

Proof. Let $\left\{x^{s}\right\}$ be a sequence in $h\left\{M_{k}, q, C\right\}$ such that $\| x^{s}-$ $x \| \rightarrow 0$, where $x \in \ell\left\{M_{k}, q, C\right\}$.

To complete the proof we need to show that $x \in$ $h\left\{M_{k}, q, C\right\}$; that is,

$$
\sum_{k \geq 0}\left[M_{k}\left(q\left(\frac{\sum_{j=0}^{k} x_{j}}{\rho(k+1)}\right)\right)\right]<\infty \quad \text { for every } \rho>0 .
$$

To $\rho>0$ there corresponds an $l$ such that $\left\|x^{l}-x\right\| \leq \rho / 2$. Then using convexity of each $M_{k}$,

$$
\begin{aligned}
\sum_{k \geq 0}\left[M_{k}\left(q\left(\frac{\sum_{j=0}^{k} x_{j}}{\rho(k+1)}\right)\right)\right] \\
=\sum_{k \geq 0}\left[M_{k}\left(q\left(\frac{2 \sum_{j=0}^{k} x_{j}^{l}-2\left(\sum_{j=0}^{k} x_{j}^{l}-\sum_{j=0}^{k} x_{j}\right)}{2 \rho(k+1)}\right)\right)\right] \\
\leq \frac{1}{2} \sum_{k \geq 0}\left[M_{k}\left(q\left(\frac{2 \sum_{j=0}^{k} x_{j}^{l}}{\rho(k+1)}\right)\right)\right] \\
+\frac{1}{2} \sum_{k \geq 0}\left[M_{k}\left(q\left(\frac{2 \sum_{j=0}^{k}\left(x_{j}^{l}-x_{j}\right)}{\rho(k+1)}\right)\right)\right] \\
\leq \frac{1}{2} \sum_{k \geq 0}\left[M_{k}\left(q\left(\frac{2 \sum_{j=0}^{k} x_{j}^{l}}{\rho(k+1)}\right)\right)\right] \\
+\frac{1}{2} \sum_{k \geq 0}\left[M_{k}\left(q\left(\frac{2 \sum_{j=0}^{k}\left(x_{j}^{l}-x_{j}\right)}{\left\|x^{l}-x\right\|(k+1)}\right)\right)\right] .
\end{aligned}
$$

Now from Theorem 9, using the definition of norm $\|\cdot\|$, we have

$$
\sum_{k \geq 0}\left[M_{k}\left(q\left(\frac{2 \sum_{j=0}^{k}\left(x_{j}^{l}-x_{j}\right)}{\left\|x^{l}-x\right\|(k+1)}\right)\right)\right] \leq 1
$$

It follows that

$$
\sum_{k \geq 0}\left[M_{k}\left(q\left(\frac{\sum_{j=0}^{k} x_{j}}{\rho(k+1)}\right)\right)\right]<\infty \quad \text { for every } \rho>0 .
$$

Thus $x \in h\left\{M_{k}, q, C\right\}$.

Hence we have the following corollary.

Corollary 16. The space $h\left\{M_{k}, q, C\right\}$ is a BK-space. 


\section{Acknowledgment}

This research is supported by Deanship of Scientific Research, Taibah University, Almadinah Almunawwarah, Saudi Arabia.

\section{References}

[1] H. Nakano, "Concave modulars," Journal of the Mathematical Society of Japan, vol. 5, pp. 29-49, 1953.

[2] J. Lindenstrauss and L. Tzafriri, "On orlicz sequence spaces," Israel Journal of Mathematics, vol. 10, no. 3, pp. 379-390, 1971.

[3] J. Y. T. Woo, “On modular sequence spaces," Studia Mathematica, vol. 48, pp. 271-289, 1973.

[4] H. Nakano, "Modulared sequence spaces," Proceedings of the Japan Academy, vol. 27, pp. 508-512, 1951.

[5] M. A. Krasnoselskii and Y. B. Rutitsky, Convex Functions and Orlicz Spaces, Groningen, The Netherlands, 1961.

[6] Ç. A. Bektaş and Y. Altin, "The sequence space $\ell_{M}(p, q, s)$ on seminormed spaces," Indian Journal of Pure and Applied Mathematics, vol. 34, no. 4, pp. 529-534, 2003.

[7] S. D. Parasar and B. Choudhary, "Sequence spaces defined by Orlicz functions," Indian Journal of Pure and Applied Mathematics, vol. 25, no. 4, pp. 419-428, 1994.

[8] M. Mursaleen, A. Khan, and Qamaruddin, "Difference sequence spaces defined by Orlicz functions," Demonstratio Mathematica, vol. 1, pp. 145-150, 1999.

[9] H. Dutta and F. Başar, "A generalization of Orlicz sequence spaces by Cesàro mean of order one," Acta Mathematica Universitatis Comenianae, vol. 80, no. 2, pp. 185-200, 2011.

[10] H. Dutta and T. Bilgin, "Strongly $\left(\left(V^{\lambda}, A, \Delta_{(v m)}^{n}, p\right)\right)$-summable sequence spaces defined by an Orlicz function," Applied Mathematics Letters, vol. 24, no. 7, pp. 1057-1062, 2011.

[11] V. Karakaya and H. Dutta, "On some vector valued generalized difference modular sequence spaces," Filomat, vol. 25, no. 3, pp. 15-27, 2011.

[12] B. C. Tripathy and H. Dutta, "Some difference paranormed sequence spaces defined by Orlicz functions," Fasciculi Mathematici, vol. 42, pp. 121-131, 2009.

[13] I. H. Jebril, "A generalization of strongly Cesàro and strongly lacunary summable spaces," Acta Universitatis Apulensis, vol. 23, pp. 49-61, 2010.

[14] K. Zeller, Theorie Der Limitierungsverfahren, Berlin, Germany, 1958. 


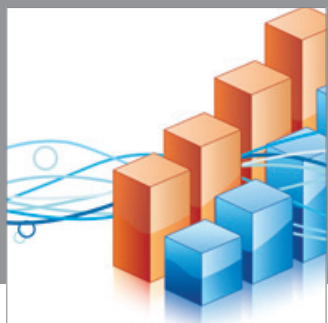

Advances in

Operations Research

mansans

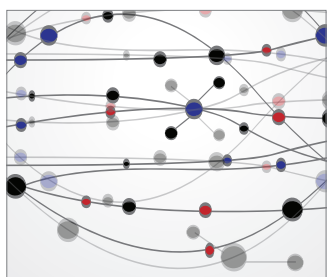

The Scientific World Journal
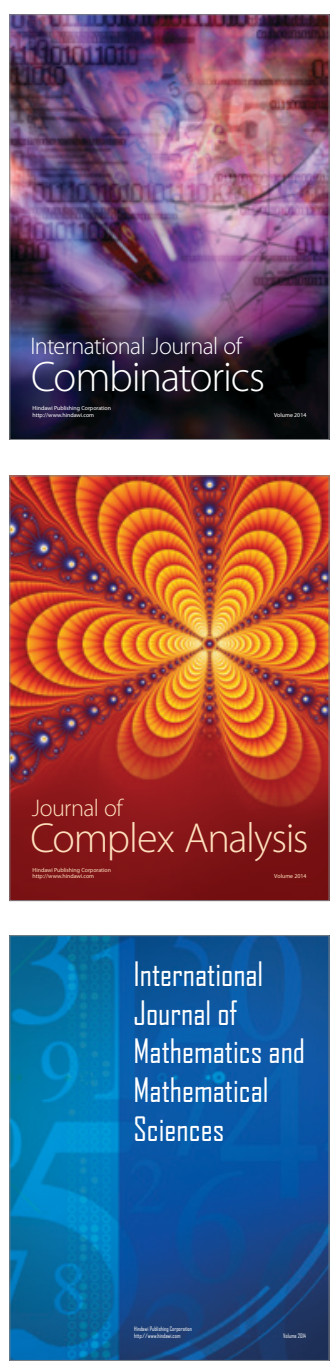
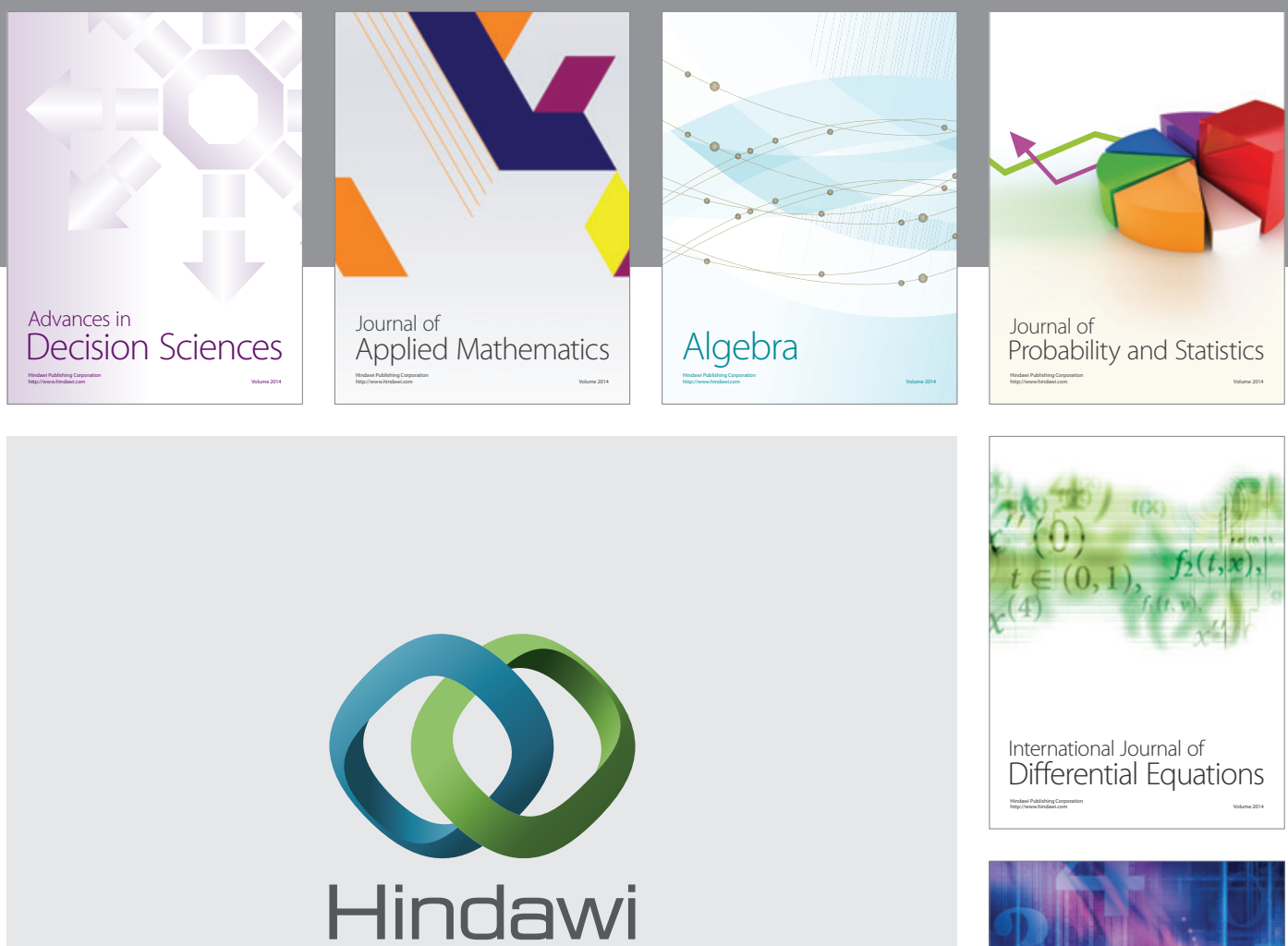

Submit your manuscripts at http://www.hindawi.com
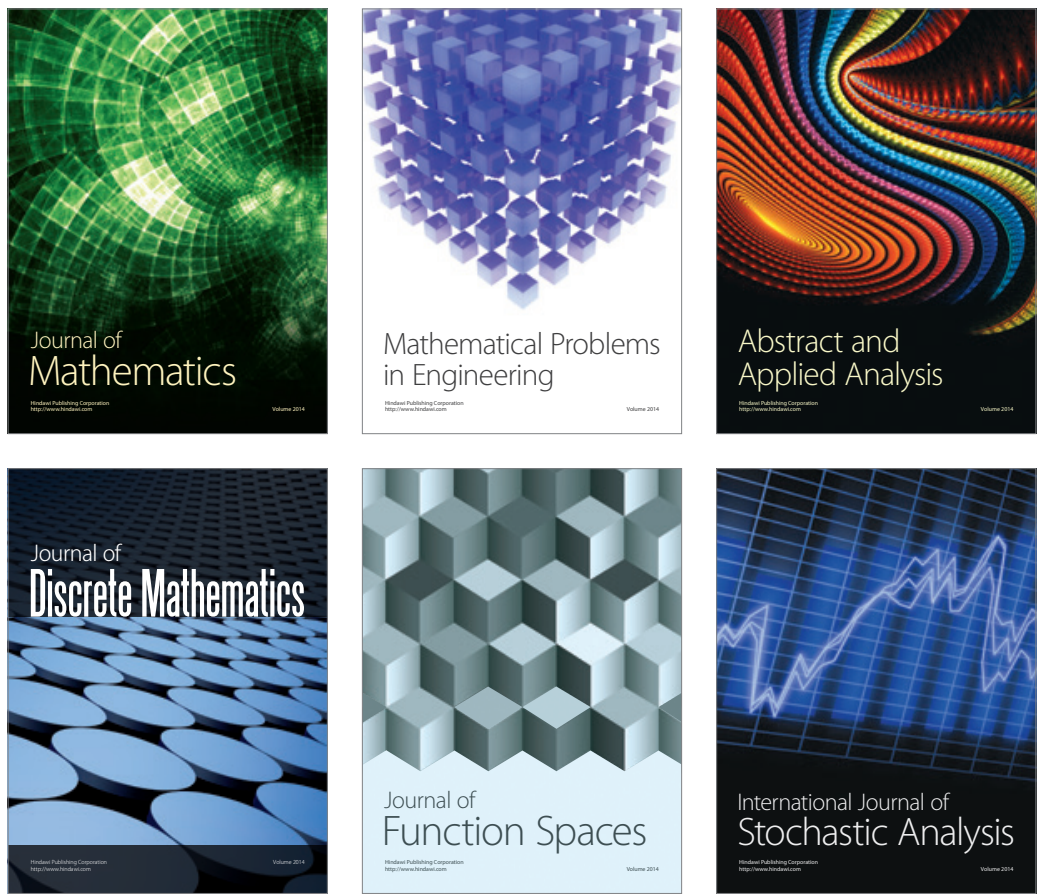

Journal of

Function Spaces

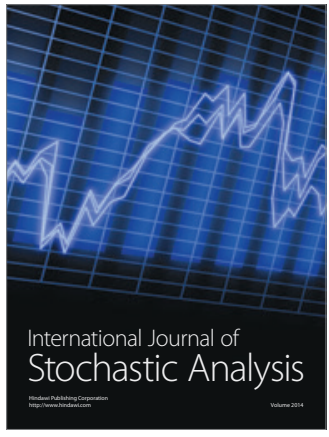

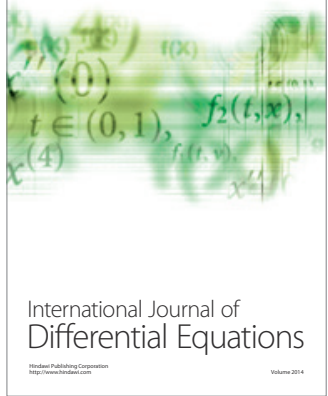
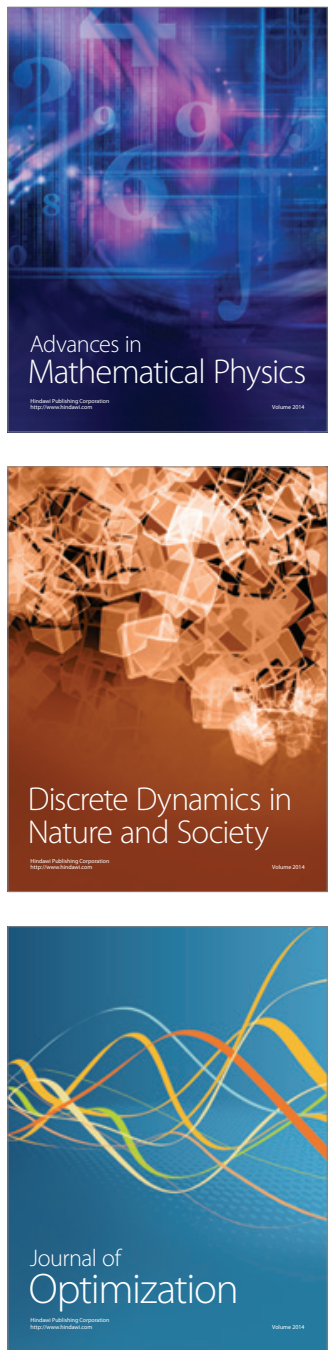